\title{
Personalized life expectancy and treatment benefit index of antiretroviral therapy
}

\author{
Yanni Xiao ${ }^{1}$, Xiaodan Sun ${ }^{1 *}$, Sanyi Tang ${ }^{2}$, Yicang Zhou ${ }^{1}$, Zhihang Peng ${ }^{3}$, Jianhong Wu ${ }^{4}$ and Ning Wang ${ }^{5}$
}

\section{${ }^{*}$ Correspondence:}

xiaodansun@mail.xjtu.edu.cn

'Department of Applied

Mathematics, Xi'an Jiaotong

University, Xianning West Road, 710049 Xi'an, China

Full list of author information is available at the end of the article

\section{Background}

Human immunodeficiency virus (HIV), the pathogen causing acquired immune deficiency syndrome (AIDS), exhibits highly complex interaction with human immune system [1, 2]. HIV infection typically results in a vast virus replication during the acute infection phase that is followed by a chronic phase where the viral load approaches a much lower quasi-steady state, and then followed by a sharp and sudden rise of viral loads when the immune system collapses [3-7]. Typical stages of HIV infection are well documented [6, 8], and the antiretroviral therapy (ART) is shown to be effective in slowing down the progression to AIDS and improving the life quality of HIV patients [9-11]. Most existing models however failed to describe the entire HIV disease progression trajectory

(c) The Author(s). 2017 Open Access This article is distributed under the terms of the Creative Commons Attribution 4.0 International License (http://creativecommons.org/licenses/by/4.0/), which permits unrestricted use, distribution, and reproduction in any medium, provided you give appropriate credit to the original author(s) and the source, provide a link to the Creative Commons license, and indicate if changes were made. The Creative Commons Public Domain Dedication waiver (http:// creativecommons.org/publicdomain/zero/1.0/) applies to the data made available in this article, unless otherwise stated. 
partly, especially they could not model the significant increase of viral loads after the development of AIDS.

There has been substantial progress in modelling antiretroviral intervention, with particular success in predicting long-term viral dynamics [12-17]. A challenge in describing the entire HIV disease progression trajectory arises from the temporal variability of the infection rate and the viral reproduction rate $[13,16,18]$. One purpose of this study is to propose a novel viral dynamic model which can describe a typical disease progression including acute infection, chronic latency and AIDS stage on the basis of the classic viral dynamic model frame $[8,19-21]$. We then show that parametrizing the infection rate and viral reproduction rate through three key parameters in the Weibull function $[22,23]$ permits us to extend the classical viral dynamics model in such a way that accurate description of the viral dynamics during the entire HIV disease progression within a host is possible. We also demonstrate, using a longitudinal cohort study in China, how parameters of the relevant Weibul functions can be estimated by fitting the viral dynamics model prediction to patient data, and how these parameterized Weibul functions in combination with the viral dynamics model yields important information about the comprehensive effects of ART on the life expectancy (LE).

Estimating the LE is important to inform the patients of their prognosis at the individual level, and to predict the future demographic and socioeconomic impact of HIV/AIDS at the population level. Several studies have investigated the prolonged LE of patients due to ART in high-income countries or resource-constrained settings at the population level [24-28], using observed mortality rates in various cohort studies. There are many challenges in determining the timing of infection, predicting the LE of HIV infected individuals and quantifying the comprehensive effects of ART on life expectancy. In the study here, based on parametrized temporal variability of infection rate and viral reproduction rate through the Weibul function which are incorporated in the classical viral dynamics model, our another purpose is to establish a predictive formula at the individual level for the LE of patients receiving ART, and to simulate how this individual LE is related to ART treatment specifics such as drug efficacy, sensitivity, adherence, treatment starting time and the time of emergence of drug resistant virus variants.

\section{Methods}

The model Let $T(t), T^{*}(t)$ and $V(t)$ be the concentrations of uninfected target CD4 T cells, productively infected cells, and free virus at time, respectively. We adopt the classic HIV viral dynamics model [19, 21], but include the temporal variability of infection rate and viral production rate in order to provide a faithful account of the entire disease progression of within an HIV patient. Namely, we have

$$
\left\{\begin{array}{l}
\frac{d T}{d t}=s-d T-k(t) V T \\
\frac{d T^{*}}{d t}=k(t) V T-\delta T^{*} \\
\frac{d V}{d t}=\lambda(t) T^{*}-c V
\end{array}\right.
$$

where $s$ is the rate of recruitment of uninfected cells, $d$ and $\delta$ are the death rates of uninfected cells and infected cells respectively, $c$ is the rate of clearance. We propose to link the temporally varying infection rate $k(t)$ and viral production rate $\lambda(t)$, in the absence of ART, to the Weibull function 


$$
W\left(t, T_{m}, \beta, \alpha\right)=1-\exp \left[-\left(\frac{T_{m}-t}{\beta}\right)^{\alpha}\right], t<T_{m},
$$

with respective location parameter $\left(T_{m}\right)$, the shape parameter $(\alpha)$, and the scale parameter $(\beta)$. The Weibull function is characterized by shape, scale and location parameters. The location parameter determines the maximum life span of the patient since infection. Using this local parameter, we can define and calculate a summative index, the treatment benefit index (TBI), as the difference of the LEs of a patient with and without ART, to measure the overall benefit of ART treatment in terms of the life year gained. Specifically, we have

$$
k(t)=\frac{k}{W\left(T_{m}, \beta_{k}, \alpha_{k}\right)}=\frac{k}{1-\exp \left[-\left(\frac{T_{m}-t}{\beta_{k}}\right)^{\alpha_{k}}\right]}, t<T_{m},
$$

and

$$
\lambda(t)=\frac{\lambda}{W\left(T_{m}, \beta_{\lambda}, \alpha_{\lambda}\right)}=\frac{\lambda}{1-\exp \left[-\left(\frac{T_{m}-t}{\beta_{\lambda}}\right)^{\alpha_{\lambda}}\right]}, t<T_{m} .
$$

with normalized constants $k$ and $\lambda$. Definitions of variables and parameters as well as the

\begin{tabular}{|c|c|c|c|}
\hline Variables & Definitions & Initial & Reference \\
\hline$T$ & Uninfected $\mathrm{CD} 4^{+}$cell population size & $1200 \mu /^{-1}$ & \\
\hline$T^{*}$ & Infected $\mathrm{CD}^{+}{ }^{+}$helper cell population size & 0 & \\
\hline$V_{1}$ & HIV population size & $100 \mu /^{-1}$ & \\
\hline$\tau$ & Prolonged LE & interim variable & \\
\hline Parameters & & baseline values [ranges] & \\
\hline s & Rate of supply of $\mathrm{CD} 4^{+} \mathrm{T}$ cell from precursors & $15 \mu l^{-1}$ day $^{-1}$ & [21] \\
\hline$d$ & Death rate of uninfected $\mathrm{CD}^{+}{ }^{+} \mathrm{T}$ cells & 0.02 day $^{-1}$ & [21] \\
\hline$k(\bar{k})$ & Infection rate per virion & $2.1818 \times 10^{-7} \mu I^{-1}$ day $^{-1}(I k)$ & [21] \\
\hline$\delta$ & Death rate of infected $C D 4^{+} \mathrm{T}$ cells & 0.35 day $^{-1}[0.20 .6]$ & [21] \\
\hline$\lambda(\bar{\lambda})$ & Number of free virus produced by lysing a $\mathrm{CD} 4^{+} \mathrm{T}$ cell & $3928.6 \mu /^{-1}$ day $^{-1}(\mid \lambda)$ & [21] \\
\hline c & Death or clearance rate of free virus & 2.4 day $^{-1}[1.53 .5]$ & [21] \\
\hline$\beta_{k}\left(\bar{\beta}_{k}\right)$ & Scale parameter of Weibull function & $1500(225)[50-2000]$ & see text \\
\hline$\beta_{\lambda}\left(\bar{\beta}_{\lambda}\right)$ & Scale parameter of Weibull function & $200(200)[10-500]$ & see text \\
\hline$\alpha_{k}\left(\bar{\alpha}_{k}\right)$ & Shape parameter of Weibull function & $1.1(1.1)[0.2-2]$ & see text \\
\hline$\alpha_{\lambda}\left(\bar{\alpha}_{\lambda}\right)$ & Shape parameter of Weibull function & $0.04(0.04)[0.005-0.2]$ & see text \\
\hline$T_{m}$ & LE since infection without therapy & $11[5,16] \times 365$ days & [34] \\
\hline$\eta(\bar{\eta})$ & Drug efficacy of combination therapy & $0.95[0.51](9 \eta)$ & {$[21]$} \\
\hline$\tau_{50}\left(\bar{\tau}_{50}\right)$ & Drug sensitivity of combination therapy & $30[20,100] \times 365$ days $\left(p \tau_{50}\right)$ & see text \\
\hline$\tau_{m}$ & Maximum LE after infection & $\left(T_{d}-T_{s}\right)$ days & - \\
\hline$T_{d}$ & Time of natural death & $74 \times 365$ days & [37] \\
\hline$T_{s}$ & Time to initiate treatment after infection & Determined by $B_{C D 4}$ & - \\
\hline$T_{r}$ & Time of emergence of drug resistant virus & Random variable in $\left[T_{s}, T_{e}\right]$ & - \\
\hline$T_{e}$ & LE after infection with treatment & $\ln \left[T_{m}, T_{d}\right]$ & - \\
\hline$T_{1000}$ & Time to virological failure & Determined by viral loads & - \\
\hline$B_{C D 4}$ & Baseline CD4 counts to initiate the treatment & $350[100-450] \mathrm{cells} / \mu /$ & - \\
\hline$d_{a}$ & Drug adherence rate & $90 \%[50-100 \%]$ & - \\
\hline
\end{tabular}
baseline parameter values are listed in Table 1. It is interesting to note that the viral loads

Table 1 Definitions of the parameters used in the model

where $I, p, q$ are modification factors related to corresponding parameters with $I=0.935, p=3, q=1$ as baseline values. And $\bar{k}=|k, \bar{\lambda}=| \lambda, \bar{\tau}_{50}=p \tau_{50}, \bar{\eta}=q \eta$. Note that over bar represents the same parameter but with values corresponding to with ART and/or drug resistance 
within-host behave like the 'bathtub curve', which shows three stages over the life time and hence is very well depicted by the proposed mixed Weibull function.

Once ART is initiated for a patient, the disease progression will be changed with altered life span. Let function $\tau(t)$ denote the treatment benefit, referred as to the TBI in what follows. This function measures the integrated effects of ART on the patients' survival so that the location parameter in the Weibull function becomes $T_{m}+\tau(t)$ with the ART. Therefore, the infection rate and the viral reproduction rate under ART become

$$
\bar{k}(t)=\frac{\bar{k}}{W\left(T_{m}+\tau(t), \bar{\beta}_{k}, \bar{\alpha}_{k}\right)}, \bar{\lambda}(t)=\frac{\bar{\lambda}}{W\left(T_{m}+\tau(t), \bar{\beta}_{\lambda}, \bar{\alpha}_{\lambda}\right)} t<T_{e},
$$

where a parameter over-bar indicates the same parameter but now associated with ART, and $T_{e}$ is the time when the patient under ART will die and this will be further explained below. In what follows, we will write $\bar{k}=l k$ and $\bar{\lambda}=l \lambda$ for a positive constant $l$.

Following the formulation of the $E_{\max }$ model $[16,29,30]$, we define TBI as the saturated function that tracks the LE of the patient at any given time $t$ :

$$
\tau(t)=\left\{\begin{array}{l}
\frac{\tau_{m} \eta\left(t-T_{s}\right)}{\tau_{50}+\eta\left(t-T_{s}\right)}, \quad T_{s} \leq t<T_{r}, \\
\frac{\tau_{m} \bar{\eta}\left(t-T_{s}\right)}{\bar{\tau}_{50}+\bar{\eta}\left(t-T_{s}\right)}+\tau_{T_{r}}, \quad t \geq T_{r} .
\end{array}\right.
$$

This function links the TBI to the drug efficacy $(\eta)$, drug sensitivity (represented by $\tau_{50}$ ), ART initiation time $\left(T_{s}\right)$ which is determined by the baseline CD4 T cell counts, time of emergence of drug-resistant virus variants $\left(T_{r}\right)$ and drug adherence (DA). We refer to Fig. 1 for various times in the entire disease progression with and without ART, where $T_{b}$ is the time of birth, $T_{i}$ is the time of infection with HIV, $T_{1000}$ is the time of virological failure after the initiation of ART, $T_{e}$ is the death time of the HIV infected individual with ART and $T_{d}$ is the time of natural death of humans given no HIV infection.

Here and in what follows, $\tau_{m}$ denotes the maximum LE since ART initiating, which can be as long as the natural LE of an uninfected individual. $\tau_{50}$ is the duration of treatment

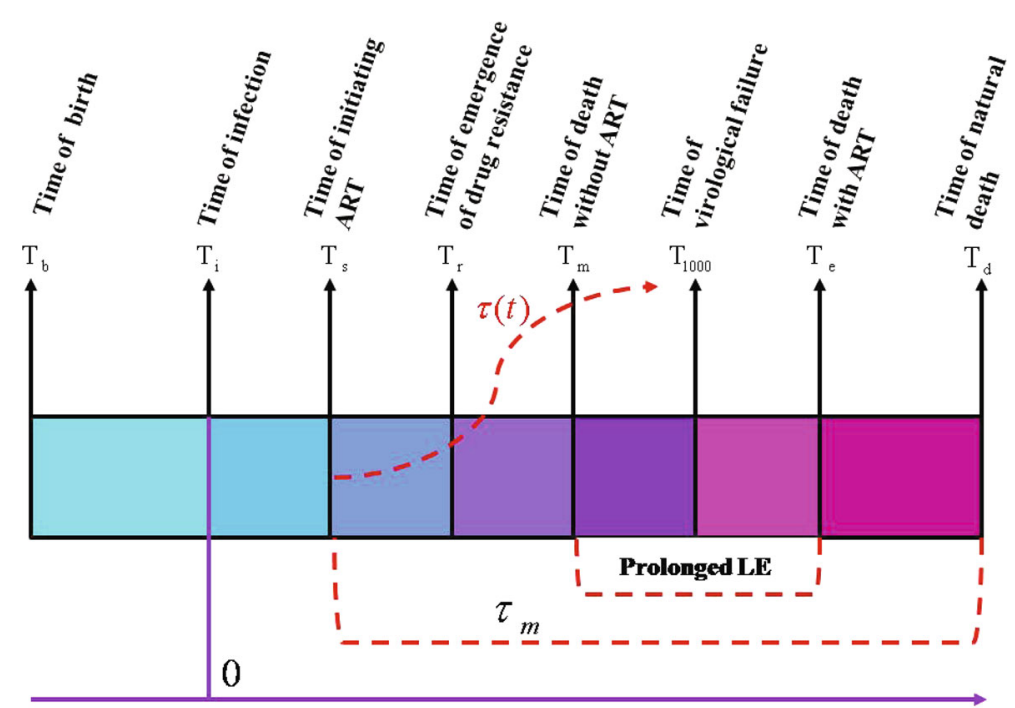

Life expectancy (LE) with first-line ART

Fig. 1 Critical points from time of infection to death. Here we set the time of infection for each patient as zero, $T_{e}-T_{m}$ represents the prolonged LE due to ART, $T_{1000}-T_{s}$ shows the duration of viral suppression during which the viral load is maintained below 1000 copies/ $\mu /$ under the first line ART regimens 
that induces an effect equivalent to $50 \%$ of the maximal LE, quantifying agent-specific drug susceptibility. The constant $\tau_{T_{r}}$ in (6) is chosen so that the function $\tau(t)$ is continuous at $T_{r}$, i.e.

$$
\tau_{T_{r}}=\tau_{m}\left[\frac{\eta\left(T_{r}-T_{s}\right)}{\tau_{50}+\eta\left(T_{r}-T_{s}\right)}-\frac{\bar{\eta}\left(T_{r}-T_{s}\right)}{\bar{\tau}_{50}+\bar{\eta}\left(T_{r}-T_{s}\right)}\right] .
$$

Recall that $\bar{\eta}$ and $\bar{\tau}_{50}$ denote the reduced drug efficacy and drug sensitivity due to emergence of drug resistant variants, we have $\tau_{50}<\bar{\tau}_{50}$ and $\eta>\bar{\eta}$. Therefore, if we write $\bar{\tau}_{50}=p \tau_{50}$ and $\bar{\eta}=q \eta$, then $p \geq 1$ and $q \leq 1$. We will address the issue of DA and its impact on TBI in the following.

Determination of LE with ART It follows that both the infection rate $\bar{k}(t)$ and the viral production rate $\bar{\lambda}(t)$ become infinity at time $t$ when the following life termination equation is satisfied:

$$
T_{m}+\tau(t)-t=0, T_{m}<t<\tau_{m}+T_{m} .
$$

The smallest root of the above equation is denoted by $T_{e}$. Note that from the point view of mathematics the LE of a patient is supposed to be associated with the infinite increases of viral loads and consequently the infinity of the viral production rate. Then the smallest root of equation (8) gives the LE with ART. To calculate this smallest root, we consider the following two cases:

$$
T_{m}+\frac{\tau_{m} \eta\left(t-T_{s}\right)}{\tau_{50}+\eta\left(t-T_{s}\right)}-t=0, \quad T_{m}<t \leq T_{r}
$$

or

$$
T_{m}+\frac{\tau_{m} \bar{\eta}\left(t-T_{s}\right)}{\bar{\tau}_{50}+\bar{\eta}\left(t-T_{s}\right)}+\tau_{T_{r}}-t=0, \quad T_{r}<t<T_{d}
$$

Denote

$$
\begin{array}{ll}
B_{1}=\left(T_{m} \eta+\tau_{m} \eta-\tau_{50}+T_{s} \eta\right), & B_{2}=\left(T_{m} \tau_{50}-T_{m} T_{s} \eta-\tau_{m} T_{s} \eta\right), \\
\bar{B}_{1}=\left(\bar{T}_{m} \bar{\eta}+\tau_{m} \bar{\eta}-\bar{\tau}_{50}+T_{s} \bar{\eta}\right), & \bar{B}_{2}=\left(\bar{T}_{m} \bar{\tau}_{50}-\bar{T}_{m} T_{s} \bar{\eta}-\tau_{m} T_{s} \bar{\eta}\right), \\
\bar{T}_{m}=T_{m}+\tau_{T_{r} .} &
\end{array}
$$

Thus, solving the Eqs. (9) and (10) yields four roots

$$
t_{12}=\frac{B_{1} \pm \sqrt{B_{1}^{2}+4 \eta B_{2}}}{2 \eta}, \quad \bar{t}_{12}=\frac{\bar{B}_{1} \pm \sqrt{\bar{B}_{1}^{2}+4 \bar{\eta} \bar{B}_{2}}}{2 \bar{\eta}} .
$$

The smallest one of real roots $t_{12}$ and $\bar{t}_{12}$, lying in the interval [ $T_{m}, T_{d}$ ], is what we want to find, and denoted by $T_{e}$. Then $T_{e}-T_{m}$ gives the prolonged LE due to ART, indicates the integrated treatment benefits. Based on the feasibility of four roots we can provide the formula of $T_{e}$ and one of the possible cases is discussed in the following. Note that here $T_{d}$ is set to be equivalent to or greater than the $T_{e}$. Due to the fact the extension of the life by ART has been increasing, we then assume that the LE of patients with ART can be as long as the average $\mathrm{LE}$ of individuals without infection.

Let $B_{2} \geq 0$ and $\bar{B}_{2} \geq 0$, then we have $\Delta>0$ and $\bar{\Delta}>0$, where $\triangle=B_{1}^{2}+4 \eta B_{2}$ and $\bar{\triangle}=\bar{B}_{1}^{2}+4 \bar{\eta} \bar{B}_{2}$, then two roots $t_{1}$ and $\bar{t}_{1}$ are positive. And further, if these two roots are in their intervals, respectively, then $T_{e}$ can be defined as

$$
T_{e}=\min \left\{t_{1}, \bar{t}_{1}\right\}=\min \left\{\frac{B_{1}+\sqrt{\triangle}}{2 \eta}, \frac{\bar{B}_{1}+\sqrt{\bar{\Delta}}}{2 \bar{\eta}}\right\} .
$$


This formula shows how the drug efficacy $(\eta$ or $\bar{\eta})$, sensitivity $\left(\tau_{50}\right.$ or $\left.\bar{\tau}_{50}\right)$, time of emergence of drug resistant virus $\left(T_{r}\right)$ affect the prolonged $\mathrm{LE}$, and consequently the disease progression. In particular, $T_{e}=\left(B_{1}+\sqrt{\triangle}\right) /(2 \eta)$ indicates that the drug efficacy is quite poor and the patient dies before the emergence of drug-resistant virus; while $T_{e}=\left(\bar{B}_{1}+\sqrt{\bar{\Delta}}\right) /(2 \bar{\eta})$ indicates that drug-resistant variants emerge during ART when the patient is alive.

Based on above definitions and analyses, we can see that the natural HIV disease progression is defined in the interval $\left[T_{i}, T_{m}\right]$, and ART prolongs the LE till $T_{e}$. From the mathematical point of view, we can simulate the model (1) with (3-4) in the interval $\left[T_{i}, T_{m}\right)$ or model (1) with (5) in the interval $\left[T_{i}, T_{e}\right)$ to produce the whole disease progressions without or with ART.

Formulation of DA To describe the effects of DA on treatment benefit, we further extend the TBI to include the adherence rate $\left(d_{a}\right.$, the fraction of the prescribed doses of the drug which are actually taken), and also to include various patterns of randomly or regularly missed doses. Assuming that once patients take doses daily, which will contribute to the function TBI, while the TBI keeps at a day for doses missing. Let $D_{a}$ be a set of days when doses are missed, then we have

$$
D_{a} \subset D_{\tau_{m}}=\left\{t, T_{s} \leq t \leq T_{e}\right\} .
$$

Denote the time intervals $T^{[i]}=[i, i+1]$, with integer $i \in\left[T_{s}, T_{e}\right]$. The daily treatment benefit functions are as follows.

$$
\begin{gathered}
\tau_{i}^{\left[T_{s}, T_{r}\right)}(t)=\frac{\tau_{m} \eta\left(t-h_{i}^{1}-T_{s}\right)}{\tau_{50}+\eta\left(t-h_{i}^{1}-T_{s}\right)}, t \in T^{[i]}, T_{s} \leq i<T_{r} \text { and } i \notin D_{a}, \\
\tau_{i}^{\left[T_{r}, T_{e}\right]}(t)=\frac{\tau_{m} \bar{\eta}\left(t-h_{i}^{2}-T_{s}\right)}{\bar{\tau}_{50}+\bar{\eta}\left(t-h_{i}^{2}-T_{s}\right)}+\tau_{T_{r}}, t \in T^{[i]}, T_{r} \leq i \leq T_{e} \text { and } i \notin D_{a},
\end{gathered}
$$

where $h_{i}^{1}$ and $h_{i}^{2}$ represent the accumulative number of days before $i+1$ days, when drug doses are missed during treatment intervals $\left[T_{s}, T_{r}\right)$ and $\left[T_{r}, T_{e}\right]$, respectively. Let $\tau_{i+1}^{c_{1}}=\tau_{i}^{\left[T_{s}, T_{r}\right)}(i+1)$ or $\tau_{i+1}^{c_{2}}=\tau_{i}^{\left[T_{r}, T_{e}\right]}(i+1)$, so if the dose is missed at the first treatment day (i.e. $T_{s}$ ), then we have $\tau_{T_{s}}^{c_{1}}=0$.

Based on the above notations we can define TBI $(\tau(t))$ at $i$-th interval $T^{[i]}$ with any pattern of drug adherence as following:

$$
\tau(t)= \begin{cases}\tau_{i}^{c_{1}}, & i \in D_{a}, t \in T^{[i]} \text { and } T_{s} \leq i<T_{r}, \\ \tau_{i}^{\left[T_{s}, T_{r}\right)}, & i \in D_{\tau_{m}} \backslash D_{a}, t \in T^{[i]} \text { and } T_{s} \leq i<T_{r}, \\ \tau_{i}^{c_{2}}, & i \in D_{a}, t \in T^{[i]} \text { and } T_{r} \leq i \leq T_{e}, \\ \tau_{i}^{\left[T_{r}, T_{e}\right]}, & i \in D_{\tau_{m}} \backslash D_{a}, t \in T^{[i]} \text { and } T_{r} \leq i \leq T_{e} .\end{cases}
$$

The data We consider a longitudinal cohort study that recruited 464 HIV infected individuals from Aihui, Hubei and Yunnan provinces from November 2003 and the cohort has been followed until now. The ART information and clinical/lab biomarker data were collected, including viral loads every 6 months and CD4 T cell counts every 3 months. Due to the cost, viral loads for majority of patients were not tested for each follow-up, and hence data on viral loads are missing. We analyzed the data anonymously.

In the cohort, few patients were tested at the ART starting point, and most of patients have their first data points after a period of ART. Among these subjects, the CD4 
$\mathrm{T}$ cell counts for 94 patients were less than 350 cells/ $\mu l$ during the ART, shown in Fig. 2. There are 149 patients whose CD4 $\mathrm{T}$ cell counts rebounded within interval [350, 550], 119 patients whose CD4 $\mathrm{T}$ cell counts rebounded within interval [550, 750], and 102 patients whose CD4 $\mathrm{T}$ cell counts rebounded above 750 . Therefore, in order to parametrize our model from the data, to reconstruct individual patient's disease progressions and TBI, we focused in this study on those patients for which we have sufficient data about their CD4 $\mathrm{T}$ cell counts and viral loads. As a result, 15 patients (listed as patient's numbers between 1 to 15 ) are selected in our study, and we divide them into three groups: (G1). Four death cases (named as patients 1-4) whose CD4 counts and viral loads were tested only after a period of treatment; (G2). Three cases (patients 5-7) with ongoing first-line ART whose CD4 T cell counts and viral loads were tested at the beginning ART; (G3). Eight cases (patients 8-15) with ongoing first-line ART whose CD4 $\mathrm{T}$ cell counts and viral loads were tested after a period of treatment.

The simulation method By using the least square method and fitting the proposed model to the data together with information on first data point and/or the date of death for the death cases we estimate some model parameters which are associated with Weibull function (shape, scale and location parameters), drug sensitivity and efficacy, ART initiation time, time of emergence of drug-resistant variants and LE. Other model parameters such as the rate of supply of CD4+ T cell from precursors $s$, death rate of uninfected CD4+ T cells $d$, the baseline infection rate per virion $k$ and etc are chosen from literature and listed in Table 1. Numerical simulations for the proposed model

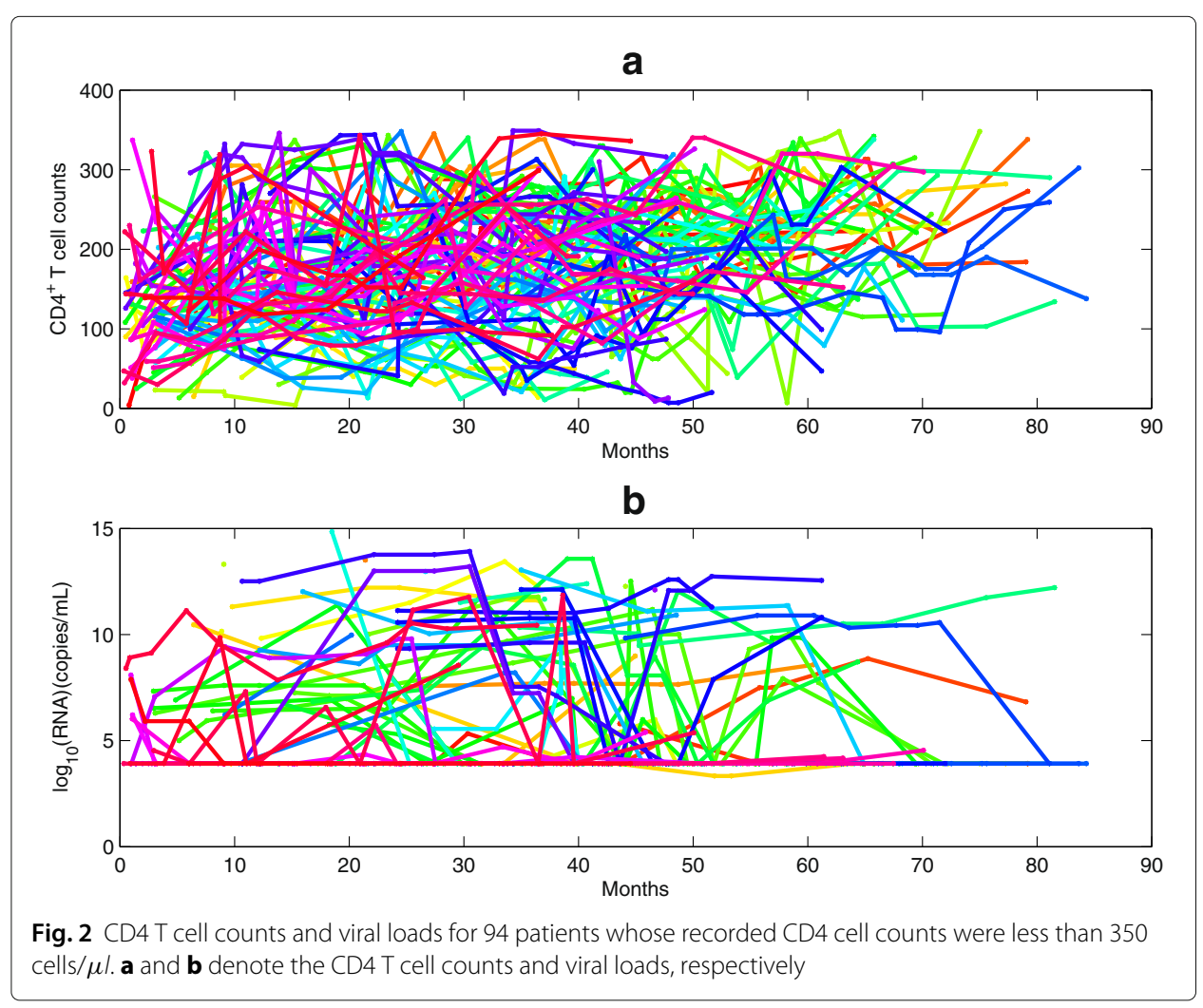


are carried out using Matlab 8, for the duration from infection $\left(T_{i}\right)$ to death $\left(T_{m}\right.$ or $T_{e}$ with ART).

\section{Results}

\section{Mimicking the entire HIV disease progression}

By linking the three-parameter Weibull functions to temporal variations of infection rate and viral production rate, we obtain a non-autonomous system for the viral dynamics during the entire disease progression within a host. Our goal is to use this non-autonomous system to examine the comprehensive effect of ART on LE including the virological failure. Using the Weibull function for the temporal variation of the infection rate $(k(t))$ and viral production rate $(\lambda(t))$ in a classic HIV viral dynamics model, we are able to produce the viral dynamics in the entire disease progression shown in Fig. 3, faithfully capturing the observed patterns (see, for example, [31]) in the early weeks of infection, during the latency and the progression to AIDS.

The Weibull function, for either infection rate or viral production rate, involves three parameters: shape, scale and location parameters. Our simulations show that each of these parameters has impact on the overall patterns of disease progression. The scale parameter $(\beta)$ governs how severe the infection is or what quasi-stationary values of the viral loads are during the latency stage (see, Fig. $4 \mathrm{a}, \mathrm{c}$ ); the shape parameter $(\alpha)$ determines how fast/slow of progression to AIDS during the late stage of disease progression, as shown in Fig. $4 \mathrm{~b}, \mathrm{~d})$; and the location parameter $\left(T_{m}\right)$ determines the survival time since infection (Fig. 5). This shows that different patients may have different patterns of disease progression even if they have the same life span $T_{m}$, and that the CD4 T cell counts and

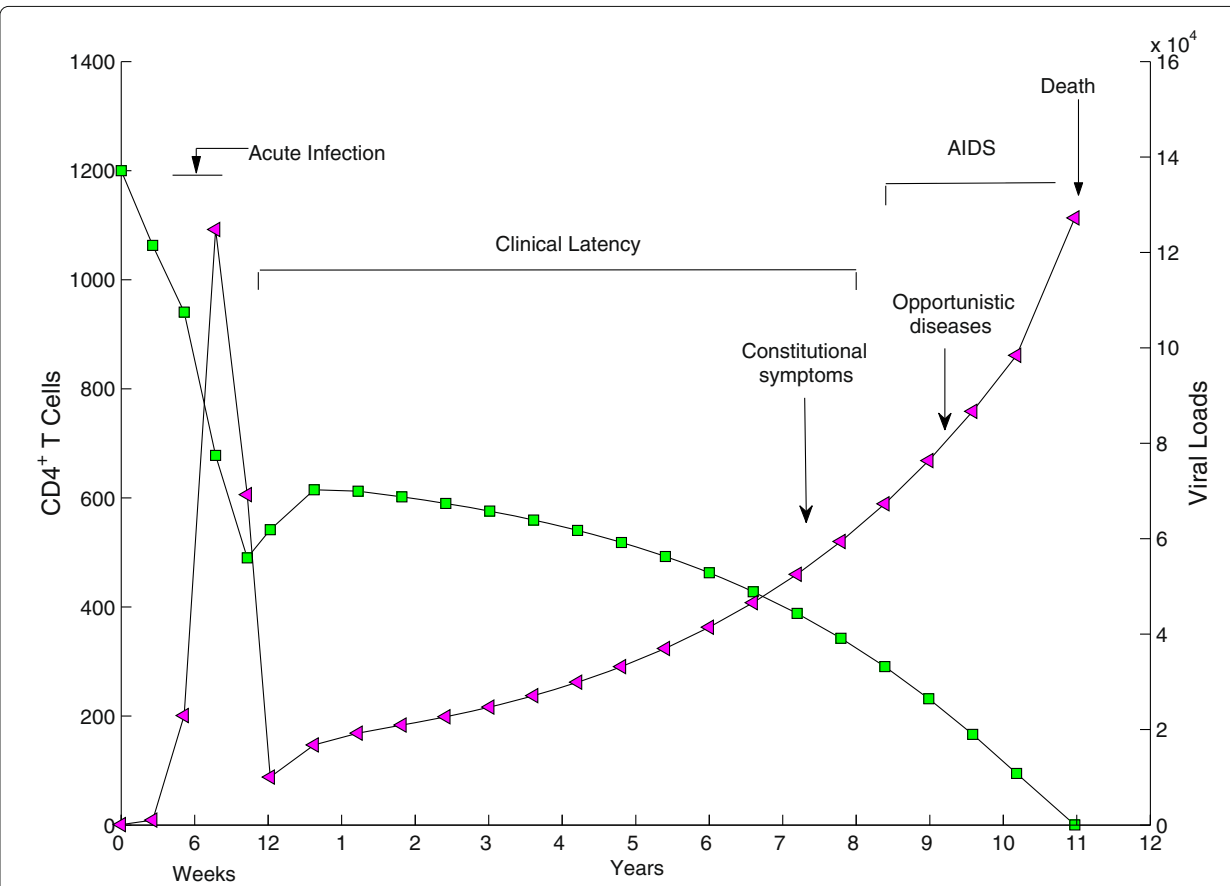

Fig. 3 A simulated typical course of HIV infection: CD4+ T cell counts and viral load. The baseline parameters are listed in Table 1 and $T_{m}=11$ years +12 weeks 


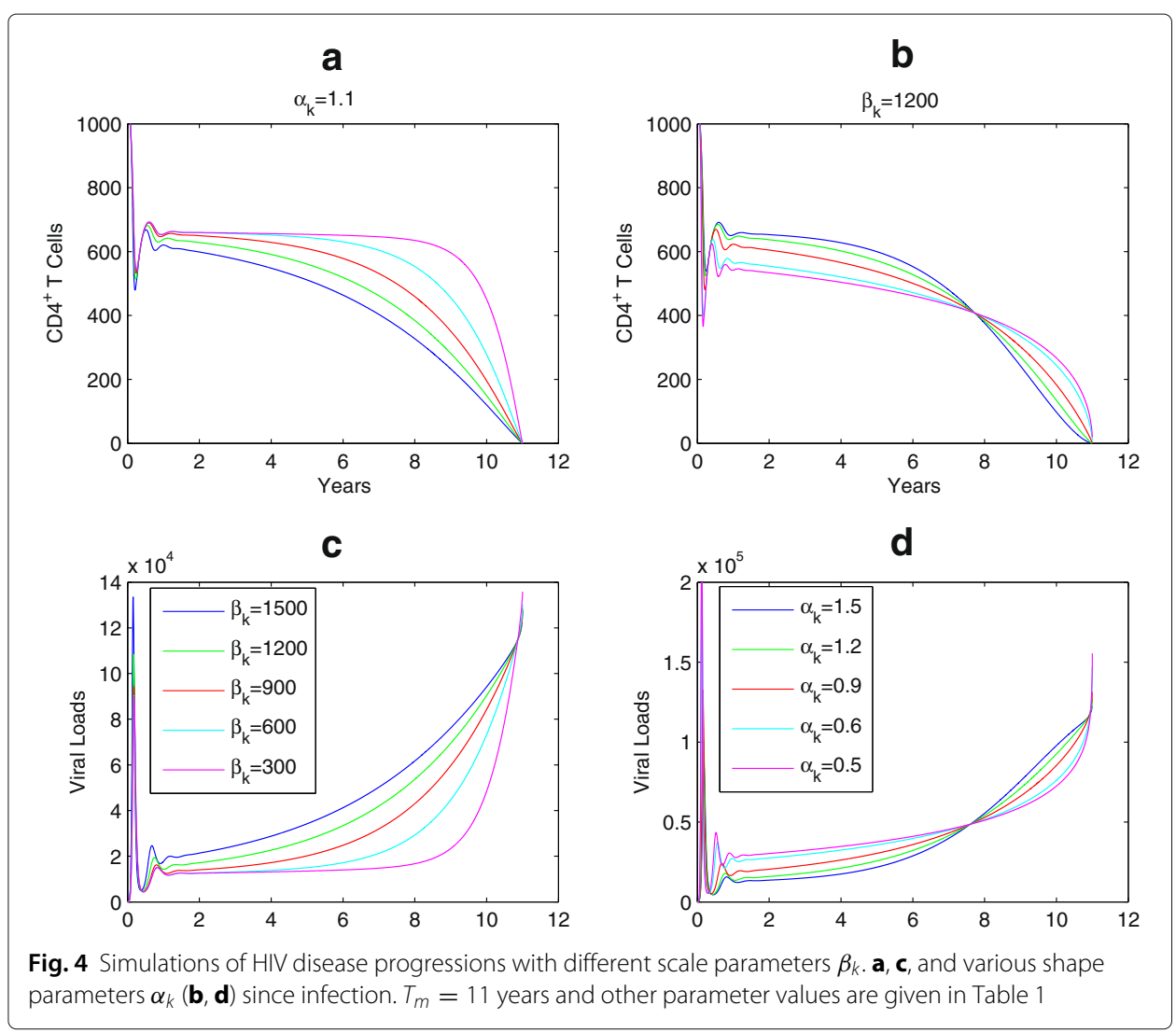

viral loads during the disease progression can be highly influenced by the values of shape and scale parameters $[6,32,33]$.

\section{Treatment benefit and LE}

We apply our model to examine the integrated effect of ART on the LE of a patient. The maximum/optimal prolonged LE is given by the difference of the $\operatorname{LE}\left(T_{e}\right)$ when the patient is on ART and the $\operatorname{LE}\left(T_{m}\right)$ when the patient is without ART, that is, $\tau_{m}=T_{e}-T_{m}$ (see critical time points in Fig. 1). The actual prolonged LE defined as the Treatment Benefit Index (TBI) at any given time $t$ since the initiation of ART $\left(T_{s}\right)$ is given by a saturated function $\tau(t)(6)$. This index summarizes the integrated effect of ART on the LE of a patient, and more importantly as will be shown in next section, this summative index can be reconstructed from the individual clinic data. The actual LE of the patient is then determined when the viral reproduction function reaches infinite, and this can be analytically calculated by finding the smallest root of a simple algebraic equation $T_{m}+\tau(t)-t=0$. As the TBI tracks the prolonged LE during the ART, it is natural to observe (simulations not reported here) that early initiation of ART delays disease progression and results in long LE, and further simulations show that late emergence of drug resistant variants or strong sensitivity leads to an increase in LE.

The viral dynamics model when the TBI is added to $T_{m}$ in the Weibull functions describes the disease progression during ART. This allows us to predict the virological failure time, the time when viral loads respond to 1000 copies/ $\mu l$ since starting ART. This model also allows us to examine how the baseline CD4 cell counts $B_{C D 4}$ (the $\mathrm{CD} 4$ cell 


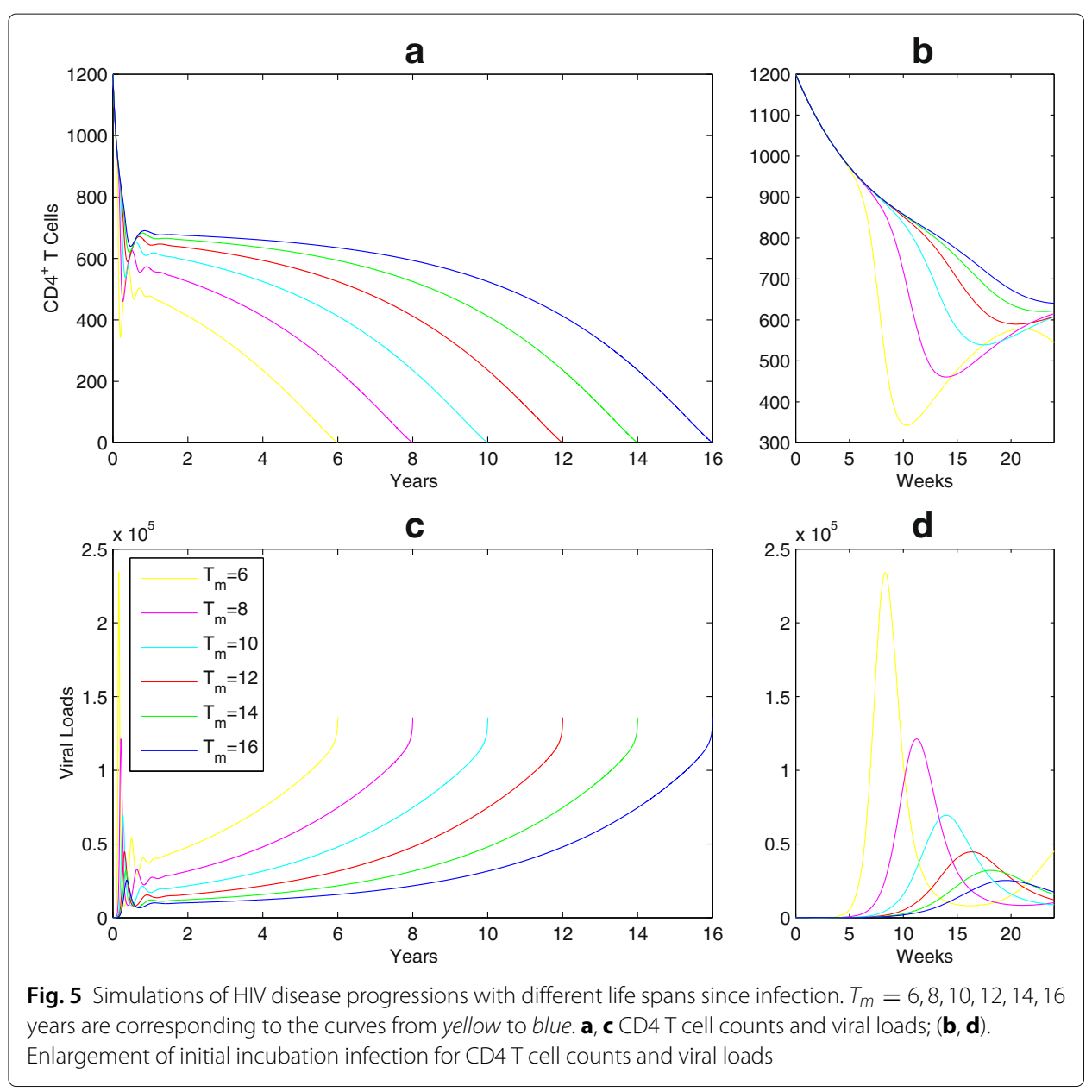

counts to initiate ART) impacts the prolonged LE and the duration of viral suppression $\left(T_{1000}-T_{s}\right)$. Figure $6 \mathrm{~b}$, for example, shows that LE increases as the baseline CD4 cell count increases. It is interesting to observe that $T_{1000}$ is not sensitive to the baseline CD4 T cell counts (Fig. 6c), for patients with the same $T_{m}$. On the other hand, Fig. $6 \mathrm{~d}-\mathrm{e}$ shows that early initiation of ART can not only increase the prolonged LE, but also prolong the duration of viral suppression. In particular, if $B_{C D 4}=350 \mathrm{copies} / \mu l, T_{m}=11$ years and other parameter values are fixed as Table 1 , then an individual who started ART at $T_{s}=7.728$ years after infection potentially has LE of $T_{e}=15.2244$ years (the prolonged LE is 4.2244 years) and the duration of viral suppression is about 2.5821 years. Our simulations also illustrate that the prolonged LE or duration of viral suppression is insensitive to variation in $T_{m}$ (Fig. 6d-f), given the baseline CD4 T cell counts.

After drug-resistant virus variants emerge, the drug sensitivity and efficacy decline [34] (with the reduction factors denoted by $p$ and $q$ in our study). Figure $7 \mathrm{~b}-\mathrm{d}$ shows that the prolonged LE and the duration of viral suppression are shortened with declining drug sensitivity. Figure $7 \mathrm{f}-\mathrm{h}$, on the other hand, shows that these durations are extended with the late emergence of drug resistant virus variants (large $T_{r}$ ). In particular, for a patient extremely (e.g. $\tau_{50}=30$ ) or normally $\left(\right.$ e.g. $\tau_{50}=80$ ) sensitive to drugs, the prolonged LE and the duration of viral suppression increase by 1.4957 and 0.9842 or 0.4518 and 0.0012 years, respectively, with one year delay of drug resistance. 


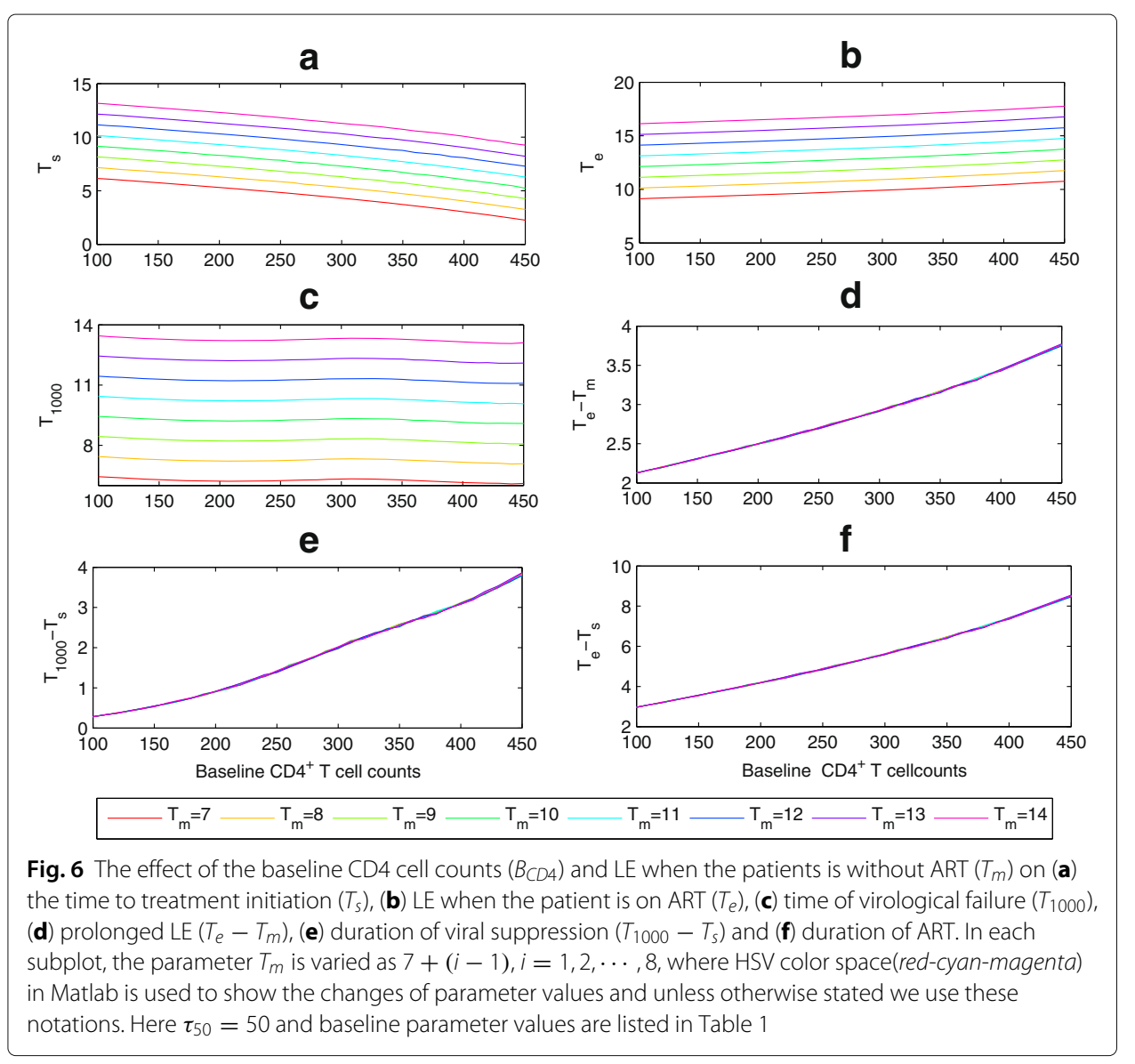

Virological failure occurs when ART fails to suppress a patient's viral loads to less than 1000 copies $/ \mu l$, and a new treatment regimen may have to be chosen to better control the infection. We further conducted simulations of disease progression, based on the proposed models with piecewise TBI, to examine the second-line regime and its contribution to LE. If the second-line regime has superior efficacy compared with the first-line regime and patients are more sensitive to the second-line drugs than the first-line ones, it could greatly prolong LE and durably suppress viral loads, as shown in Fig. 8. This is in agreement with the finding that second-line ART in South Africa achieved durable viral suppression in three-quarters of patients [24]. However, if the second-line drugs have the relatively similar efficacy to the first-line ones, it barely suppresses viral reproduction but still can prolong the LE. This indicates that the second-line drugs should be more effective than the first-line one in order to maximize the LE of a patient.

\section{Effect of adherence on drug efficacy}

Simulating our proposed model with function $\tau(t)$ defined (17) shows that the HIV disease progresses slowly with hight rate of adherence (not reported here). To show the effects of different DA patterns on the HIV disease progression, we consider the following two special patterns:

Case 1: Random pattern Suppose does missing is a random event due to uncertainty, we randomly take the days from the interval $\left[T_{s}, T_{e}\right]$ with a proportion of $1-d_{a}$. We 

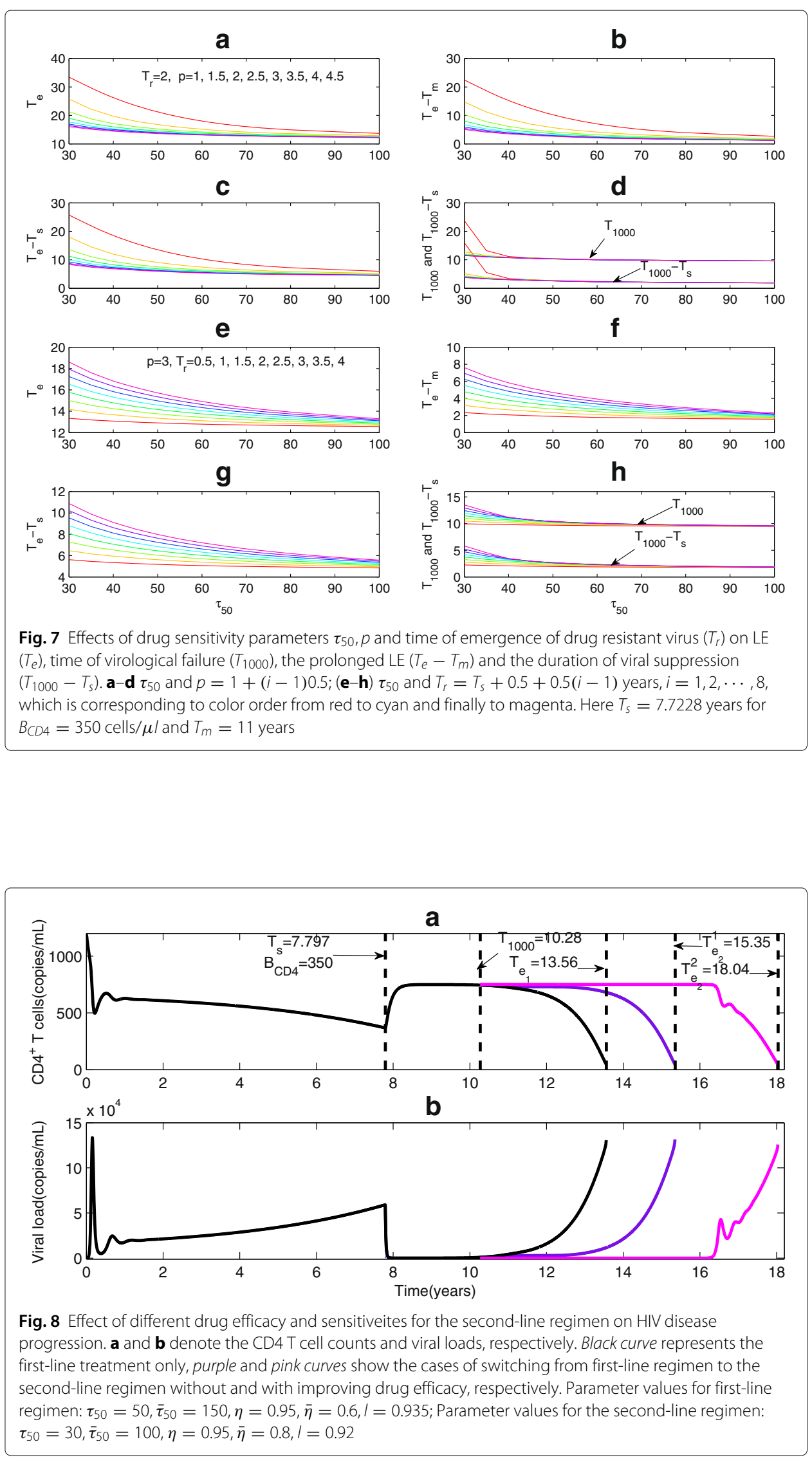
run the simulations with various DA rates and again obtain that the greater DA rate the longer mean LE.

Case 2: Regular pattern Let $w_{1}\left(w_{2}\right)$ be the numbers of days of drug-on (drug-off), then DA rate yields $d_{a}=\frac{w_{1}}{w_{2}+w_{1}}$. Therefore, we call the regular pattern as $n w_{1}: n w_{2}$ pattern with $n=1,2,3, \cdots$, where $n$ depicts the frequency of on-off pattern switching with fixed adherence rate $d_{a}$.

A cohort study in China [34] has indicated that imperfect DA is an important factor that reduces drug efficacy and sensitivity. Thus, in order to depict this point, we assume the drug efficacy and sensitivity are functions of $h_{i}^{2}$, the dynamic accumulative days with missed doses during treatment intervals $\left[T_{s}, T_{e}\right]$. So we have the revised drug efficacy and sensitivity as the following:

$$
\tau_{50}^{D A}(t)\left(\bar{\tau}_{50}^{D A}(t)\right)=\tau_{50} r_{1}^{h_{i}^{2}}\left(\bar{\tau}_{50} r_{1}^{h_{i}^{2}}\right), \eta^{D A}(t)\left(\bar{\eta}^{D A}(t)\right)=\eta r_{2}^{h_{i}^{2}}\left(\bar{\eta} r_{2}^{h_{i}^{2}}\right), t \in T^{[i]}
$$

with $r_{1}>1$ and close to $1, r_{2}<1$ and close to 1 . Since no reliable information of how DA influences on the time of emergence of resistant virus variants, we simply do not consider the effects of DA on time of emergence of resistant strain here.

When the pattern of patient's missed doses is regular, we can use the model to examine the effect of frequency of drug on-off switching on LE. Figure 9 shows the distributions of LE and correlation between duration of drug on $\left(w_{1}\right)$ and LE for various regular adherence patterns at a given adherence rate of $90 \%$. Figure 9 a gives the distribution of 50 simulations with various switching frequencies and Fig. 9b shows the positive correlation between

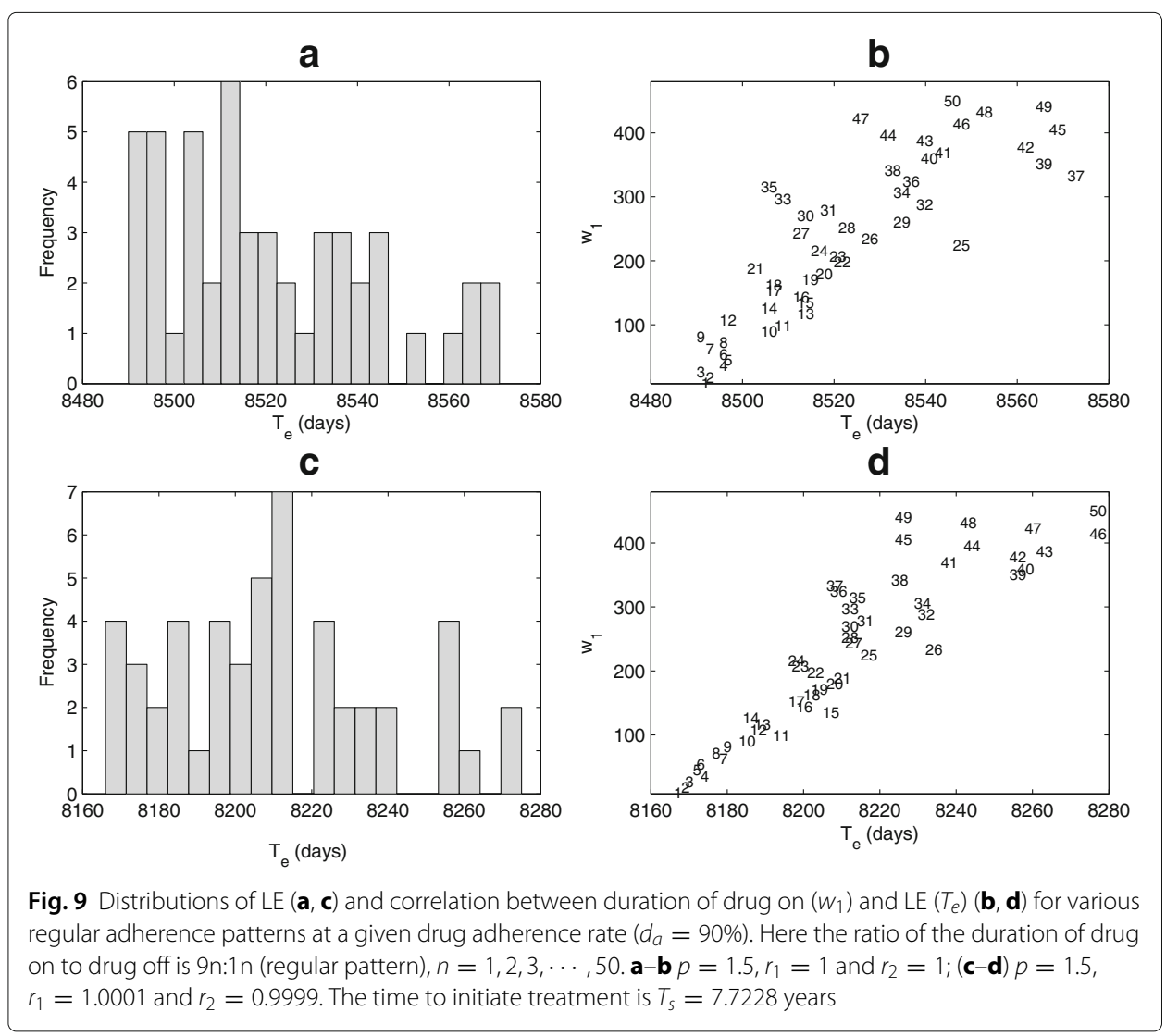


LE $\left(T_{e}\right)$ and the duration of drug on. We conclude that a long duration of drug on (and consequently a long duration of drug off due to the fixed DA rate) could yield slightly longer LE by about 100 days. A repeat of the above with low drug efficacy and decreased sensitivity due to imperfect adherence gives a short LE (shown in Fig. 9c, d).

In the case of randomly missed dose pattern, our simulations demonstrate that TBI decreases and disease progresses faster with less DA rate. The simulated distributions of LE again illustrate a comparative advantage of increasing adherence (not shown here). For a given adherence $60 \%$ (or 90\%), the mean LE is 16.6944 (or 23.2532) years with range of 271 (177) days. Similarly, lower drug efficacy and decreased sensitivity in such a scenario give a shorter mean LE.

To examine how different DA rates and dose missing patterns with various switching frequencies influence LE and the time of virological failure, we plot variation in LE with DA rates for different patterns. Figure 10a and $b$ show that the LE and prolonged LE are not sensitive to adherence patterns for a given DA rate. However, timing of virological failure $\left(T_{1000}\right)$ and duration of viral suppression $\left(T_{1000}-T_{s}\right)$ show great variance for different adherence patterns, especially for 85 and $65 \%$ adherence (shown in Fig. 10c-d). This implies that frequently switching drug on and off is not beneficial to suppress viral replication, and hence results in slight shorter LE.

\section{A case study: reconstruction of TBI from the clinical data}

Some model parameters are chosen from literature and other model parameters (such as shape, scale and location parameters, drug sensitivity and efficacy, ART initiation

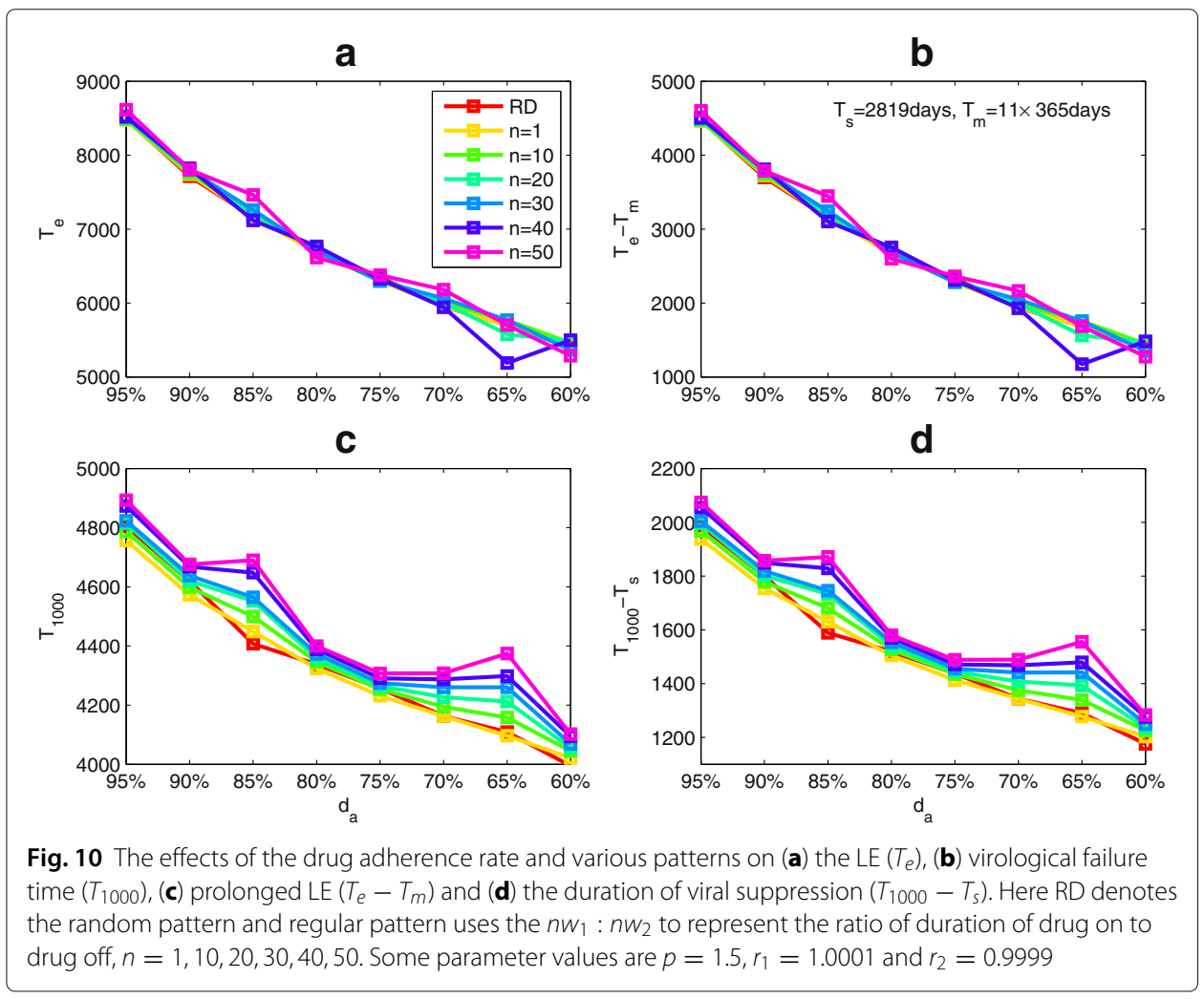


time, time of emergence of drug-resistant variants, and LE) are estimated by fitting the proposed model to the data together with information on first data point and/or the date of death for the death cases. All estimated parameter values for 15 patients are listed in the Tables 2 and 3, and the data fitting results for patient 8 to patient 11 are shown in Fig. 11 (fitting results for other patients not reported here). In summary, we observed that patients with adequate (high) CD4 response achieved relatively long prolonged LE (see patients 8 and 10 in Fig. 11), patients with poor (low) CD4 response had relatively short prolonged LE, while others whose CD4 counts barely responded to ART almost had no prolonged LE.

The TBI can be reconstructed based on the estimated parameter values for all 15 patients, as shown in Fig. 12. This predicts the prolonged LE for each individual patient on ongoing first-line regime. The turning point of each estimated TBI in Fig. 12 is the time of emergence of drug-resistant virus variants, after which drug efficacy and sensitivity are reduced. The TBI reported in Fig. 12a and estimated parameters for patient 3 show that $\operatorname{drug} \operatorname{efficacy}(\eta=0.605)$ and sensitivity $\left(\tau_{50}=383.85\right)$ are both low, and this resulted in a small and slow increase of TBI over time and very insignificant increase in the prolonged LE ( $T_{e}-T_{m}=20.96$ days). Other data fits (not reported here) further confirms that the first-line ART for patient 3 barely delayed the disease progression. For the patient 4 with the first recorded CD4 counts of 40 cells/ $\mu l$, we constructed the TBI (Fig. 12a) and calculated the prolonged LE to be 299 days $\left(T_{e}-T_{m}=299.46\right)$. Patient 2 had the longest LE on the first-line ART among the four death cases, which was associated with late emergence of drug-resistant virus variants and great drug efficacy and sensitivity. The TBI for patient 8 increased with the fastest speed and latest time of emergence of drug resistant variants (Fig. 12c). Figure 11 confirms that the disease progression of this patient was significantly delayed during ongoing first-line ART. The predicted prolonged LE for patient 8 under first-line regimen is around 11.34 years $\left(T_{e}-T_{m}=4140.4\right.$ days $)$. The TBI shown in

Table 2 Estimated parameter values for patients 1-7

\begin{tabular}{|c|c|c|c|c|c|c|c|}
\hline Par. & P1 & P2 & P3 & P4 & P5 & P6 & P7 \\
\hline$\beta_{\lambda}$ & 117.95 & 107.65 & 152.83 & 136.51 & 109.63 & 109.92 & 116.75 \\
\hline$\beta_{k}$ & 1542.68 & 1614.66 & 1873.36 & 1270.91 & 1476.14 & 1471.95 & 1606.20 \\
\hline$\alpha_{\lambda}$ & 0.32 & 0.13 & 0.44 & 0.31 & 0.08 & 0.08 & 0.04 \\
\hline$\alpha_{k}$ & 0.56 & 0.99 & 0.84 & 1.08 & 1.11 & 1.11 & 1.07 \\
\hline$T_{m}$ & 4014 & 3946.7 & 4571.1 & 3739.2 & 3995 & 4002.1 & 4019.7 \\
\hline$\eta$ & 0.78 & 0.71 & 0.60 & 0.78 & 0.89 & 0.78 & 0.96 \\
\hline$\tau_{50}$ & 117.89 & 39.34 & 383.85 & 30.23 & 52.64 & 55.92 & 31.76 \\
\hline$\tau_{m}$ & 15915 & 18055 & 4496 & 7574 & 17382 & 17277 & 20596 \\
\hline 9 & 0.84 & 0.77 & 0.89 & 0.75 & 0.78 & 0.64 & 0.65 \\
\hline$p$ & 3.72 & 8.13 & 1.23 & 13.96 & 1.89 & 2.19 & 3.14 \\
\hline $\bar{\beta}_{k}$ & 73.35 & 1614.7 & 1869.7 & 12.70 & 1294.6 & 931.08 & 217.12 \\
\hline $\bar{\alpha}_{k}$ & 0.56 & 0.99 & 0.84 & 0.80 & 1.10 & 1.11 & 1.07 \\
\hline I & 0.80 & 0.99 & 0.99 & 0.86 & 0.80 & 0.99 & 0.90 \\
\hline$T_{s}$ & 2983.6 & 3681 & 3290.4 & 3358.7 & 3719.5 & 3521 & 3839.7 \\
\hline$T_{r}$ & 3796.47 & 5070.56 & 3812.76 & 3929.77 & 5544.5 & 4424.67 & 4232.49 \\
\hline$T_{e}$ & 4279.6 & 5116 & 4592.1 & 4038.7 & 4909 & 4615.4 & 6706.3 \\
\hline$T_{d}$ & 18899.09 & 21736.01 & 7786.16 & 10932.50 & 21101.71 & 20798.12 & 24436.16 \\
\hline$B_{C D 4}$ & 200 & 100 & 200 & 50 & - & - & - \\
\hline
\end{tabular}


Table 3 Estimated parameter values for patients 8-15

\begin{tabular}{lllllllll}
\hline Par. & P8 & P9 & P10 & P11 & P12 & P13 & P14 & P15 \\
\hline$\beta_{\lambda}$ & 108.12 & 109.99 & 127.17 & 107.15 & 174.51 & 112.38 & 139.62 & 113.85 \\
$\beta_{k}$ & 1619.24 & 1444.27 & 1170.06 & 1626.31 & 1586.35 & 1490.67 & 1660.41 & 1576.88 \\
$\alpha_{\lambda}$ & 0.03 & 0.13 & 0.22 & 0.04 & 0.13 & 0.06 & 0.05 & 0.08 \\
$\alpha_{k}$ & 1.18 & 1.10 & 0.93 & 1.09 & 1.23 & 1.09 & 1.17 & 1.18 \\
$T_{m}$ & 3128.4 & 4026.7 & 3701.9 & 3118.1 & 3254.5 & 3952.6 & 4300.6 & 3220.4 \\
$\eta$ & 0.99 & 0.87 & 0.95 & 0.94 & 0.96 & 0.93 & 0.93 & 0.95 \\
$\tau_{50}$ & 30 & 30.09 & 30.71 & 53.27 & 30.22 & 55.79 & 39.33 & 65.58 \\
$\tau_{m}$ & 21897 & 18753 & 21340 & 20003 & 21530 & 17541 & 21454 & 20267 \\
$q$ & 0.55 & 0.65 & 0.88 & 0.58 & 0.90 & 0.76 & 0.53 & 0.57 \\
$p$ & 4.65 & 4.98 & 3.25 & 2.61 & 3.30 & 2.11 & 3.04 & 2.18 \\
$\bar{\beta}_{k}$ & 746.11 & 619.94 & 961.22 & 189.82 & 1164.1 & 1321.1 & 814 & 772.8 \\
$\bar{\alpha}_{k}$ & 1.18 & 1.09 & 0.87 & 1.09 & 1.13 & 0.93 & 0.66 & 1.11 \\
$I$ & 0.92 & 0.86 & 0.84 & 0.92 & 0.83 & 0.93 & 0.81 & 0.93 \\
$T_{s}$ & 2441.4 & 3498.6 & 3445.7 & 2472.7 & 2599.4 & 3002 & 3215.7 & 2569 \\
$T_{r}$ & 4526.31 & 5048.56 & 4863.17 & 2872.21 & 3906.73 & 4790.24 & 4418.25 & 4498.8 \\
$T_{e}$ & 7268.8 & 6339.7 & 6938.3 & 5054.7 & 6722.5 & 5473 & 6294.4 & 4708.4 \\
$T_{d}$ & 24338.38 & 22251.46 & 24785.25 & 22476.19 & 24129.89 & 20542.58 & 24669.66 & 22835.56 \\
$B_{C D 4}$ & 200 & 200 & 150 & - & 200 & 300 & 300 & 200 \\
\hline
\end{tabular}

where Par. represents parameter, and Pi denote Patient $i$

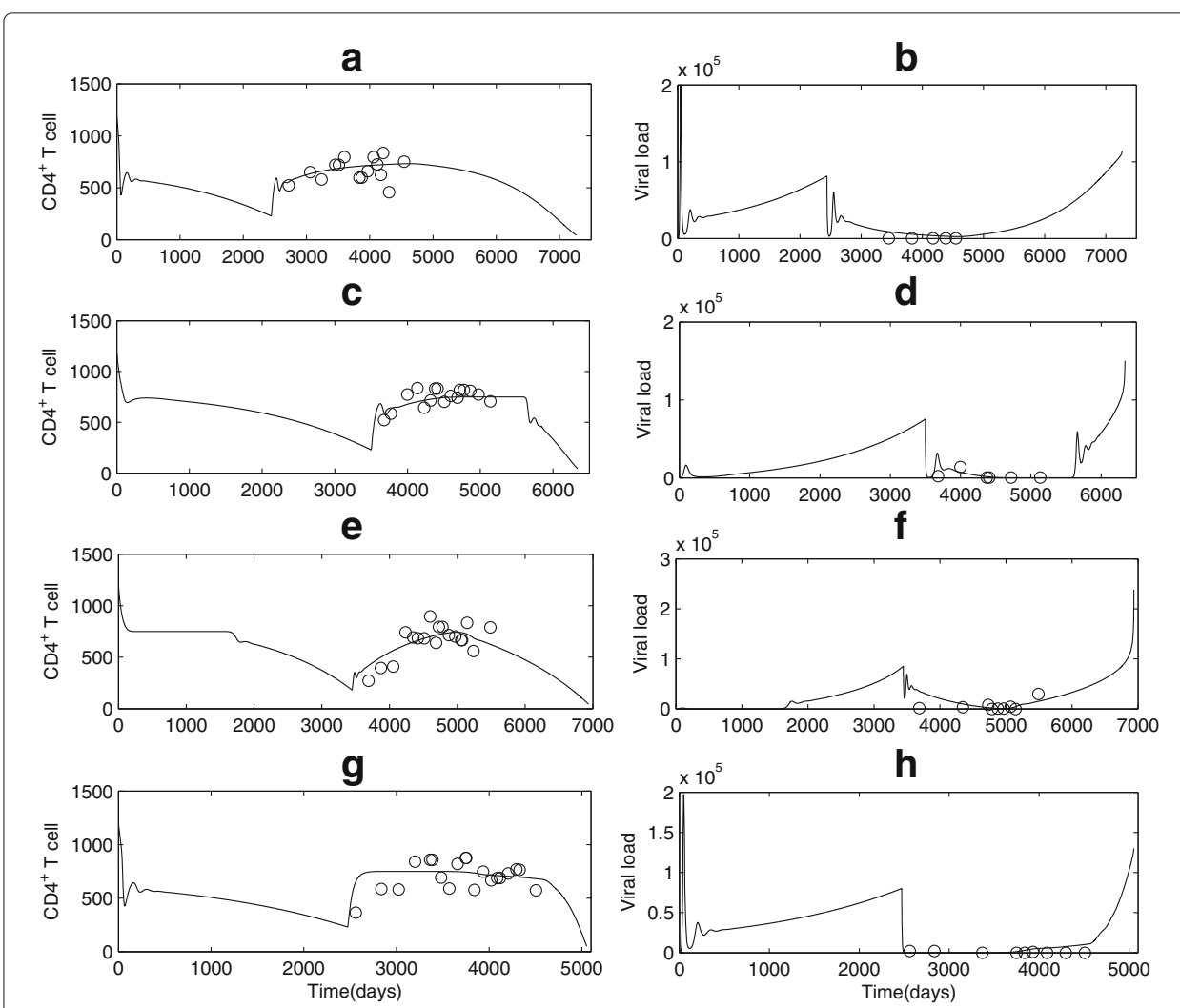

Fig. 11 Curve fitting and parameter estimation for four continuous ART cases (G3) whose CD4 count and viral load were tested after a period treatment. a-b Patient 8; (c-d) Patient 9; (e-f) Patient 10; (g-h) Patient 11 

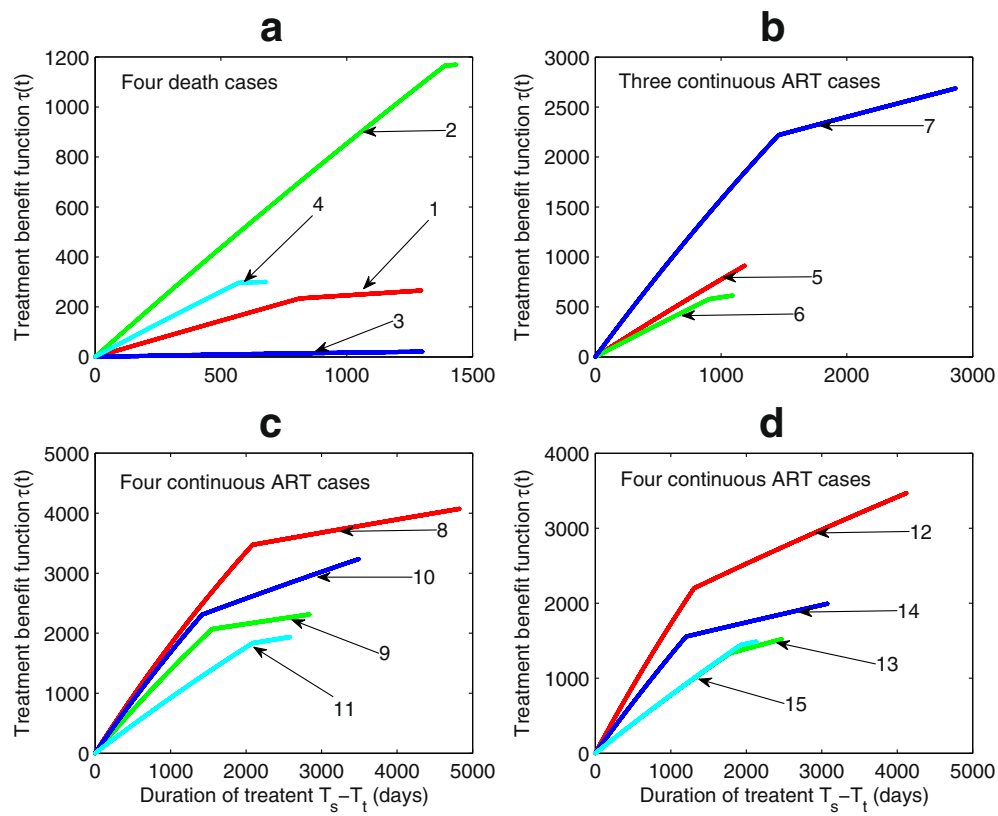

Fig. 12 Determining the TBIs for 15 patients based on parameter estimates. a TBIs for four death cases; (b) TBIs for three patients (Group 2); (c-d) TBls for eight patients (Group 3)

Fig. 12 also reveals low efficacy of the first-line treatment for patients 1, 3, 4, 5, and 6 . This further highlights the importance of early diagnosis of HIV infection and more effective ART regimens including second-line regimens should be considered for this cohort.

\section{Discussions}

It is known that HIV infection typically results in a vast replication of virus during the acute phase. The viral load then becomes much lower and approaches a quasi-steady state, and finally increases significantly after the development of AIDS [6, 35]. The viral loads change overtime and behave as the 'bathtub curve', which shows three stages over the life time and hence is very well depicted by the proposed mixed Weibull function [23] for the temporal variability of infection rate and viral reporduction rate. Despite intensive and promising progress in HIV/AIDS viral dynamics modeling, it remains a challenge to provide approximation of the entire HIV disease progression dynamics. Here, by linking the viral reproduction rate and infection rate to the Weibull function with biologically interpretable shape, location and scale parameters, we showed that the viral dynamics model can describe a typical disease progression including acute infection, chronic latency and AIDS stage. In particular, when life expectancy is assumed to be infinity, the three-parameter Weibull function becomes unity and our proposed model reduces to the classic model of HIV dynamics [8, 19, 21].

We have also shown that our model can be used to predict the LE of an HIV infected individual and the time of virological failure. The accurate description of the entire HIV disease progression makes it possible to use this model to predict the transmission probability at different stages based on viral loads, and this is important when we consider new infections generated by a particular infected individual. Our model can also be used to 
determine the timing of infection for an infected individual based on individual parameters, monitored data on CD4 cell counts and viral loads, which is difficult to get. This estimation of the infection time for each infected individuals provides vital information on estimating new infections at the population level. In addition, the knowledge about the timing of infection for HIV-infected individuals in various communities enables effective contact tracing and facilitates treatment resource allocation.

Simulating the proposed model shows early initiation of ART can result in long LE (great $\left.T_{e}\right)$ and prolonged LE $\left(T_{e}-T_{m}\right)$, in agreement with those in previous studies [26]. Since the waiting time for the emergence of resistant genomes is substantial [36] and is incorporated in our introduced TBI, we developed a continuous (rather than an impulsive model) model of HIV dynamics with switching to describe differences of drug efficacy and sensitivity after emergence of drug resistant virus variants. Our results show that later emergence of drug resistant virus variants leads to longer (prolonged) LE and more persistent viral suppression. The estimated piecewise TBIs are increasing functions with treatment duration, with a great/low slop before/after the emergence of drug resistant virus variants. Therefore, we could estimate the time of emergence of drug-resistant variants for an infected individual, which may provide information on the time for switching to the second-line regimen without resistance testing. It is known that individualized therapy is hampered by limited availability of viral load and resistance testing, making it difficult to determine whether the remaining antiviral potency of previously used drugs outweighs their toxicity [27]. Hence, our estimation makes individualized therapy more feasible and cost-effective.

\section{Conclusions}

The proposed novel modeling approach led us naturally to the introduction of the treatment benefit index (TBI) to summarize the integrated effect of ART in terms of prolonged LE. Moreover, this TBI can be reconstructed from clinical data with predicting the time of virological failure. Our model can be used to determine the timing of infection for an infected individual based on individual parameters, monitored data on CD4 cell counts and viral loads. Main results show that combining model predictions with monitored CD4 counts and viral loads can provide critical information about the disease progression, to assist the design of ART regimen for maximizing the treatment benefits.

\section{Abbreviations}

AIDS: Acquired immune deficiency syndrome; ART: Antiretroviral therapy; DA: Drug adherence LE: Life expectancy; HIV: Human immunodeficiency virus; TBI: Treatment benefit index

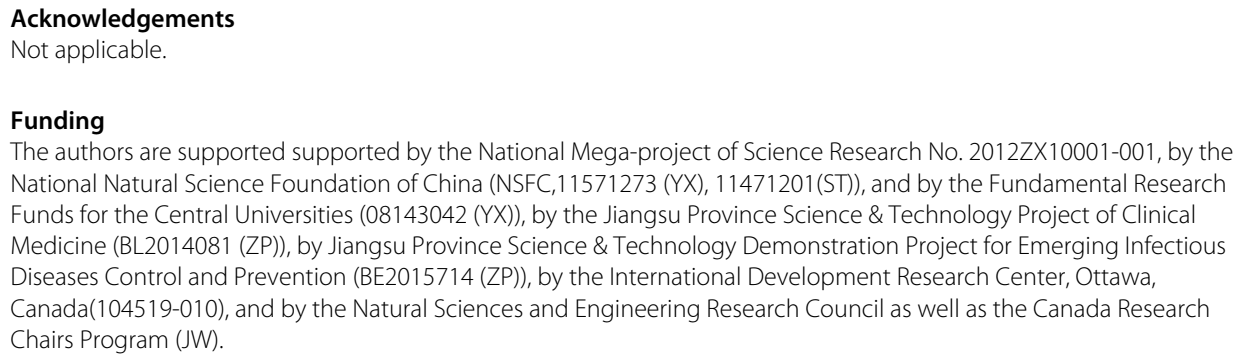

The authors are supported supported by the National Mega-project of Science Research No. 2012ZX10001-001, by the National Natural Science Foundation of China (NSFC,11571273 (YX), 11471201(ST)), and by the Fundamental Research Funds for the Central Universities (08143042 (YX)), by the Jiangsu Province Science \& Technology Project of Clinical Medicine (BL2014081 (ZP)), by Jiangsu Province Science \& Technology Demonstration Project for Emerging Infectious Diseases Control and Prevention (BE2015714 (ZP)), by the International Development Research Center, Ottawa, Canada(104519-010), and by the Natural Sciences and Engineering Research Council as well as the Canada Research Chairs Program (JW) 


\section{Authors' contributions}

NW supervised the project. YX, XS, ST, YZ, ZP, JW and NW conceived and designed the study. ZP collected the experiment data. ST and XS conducted all data analyses and simulations. YX, ST, JW and NW wrote the paper. All authors read and approved the final manuscript.

\section{Competing interests}

The authors declare that they have no competing interests.

\section{Consent for publication}

Not applicable.

\section{Ethics approval and consent to participate}

Not applicable.

\section{Author details}

${ }^{1}$ Department of Applied Mathematics, Xi'an Jiaotong University, Xianning West Road, 710049 Xi'an, China. ${ }^{2}$ College of Mathematics and Information Science, Shaanxi Normal University, West Chang'an Avenue, 710119 Xi'an, China. ${ }^{3}$ School of Public Health, Nanjing Medical University, 210029 Nanjing, China. ${ }^{4}$ Laboratory for Industrial and Applied Mathematics, Centre for Disease Modelling, York Institute for Health Research, York University, M3J 1P3 Toronto, Canada. ${ }^{5}$ National Center for AIDS/STD Prevention and Control, Chinese Center for Disease Control and Prevention, 155 Changbai Road, 102206 Beijing, China.

\section{Received: 24 September 2016 Accepted: 29 December 2016}

Published online: 18 January 2017

\section{References}

1. Nowak MA, Bangham CR. Population dynamics of immune responses to persistent viruses. Science. 1996;272:74-9.

2. De Boer RJ. Understanding the failure of CD8+ T-cell vaccination against simian/human immunodeficiency virus. J Virol. 2007;81:2838-48.

3. Wei X, Ghosh SK, Taylor ME, Johnson VA, Emini EA, et al. Viral dynamics in human immunodeficiency virus type 1 infection. Nature. 1995;373:117-22.

4. O'Brien TR, Rosenberg PS, Yellin F, Goedert JJ, et al. Longitudinal HIV-1 RNA levels in a cohort of homosexual men. J Acquir Immune Defic Syndr Hum Retrovirol. 1998;18:155-61.

5. Sabin CA, Devereux H, Phillips AN, Hill A, Janossy G, et al. Course of viral load throughout HIV-1 infection. J Acquir Immune Defic Syndr. 2000;23:172-7.

6. Ho DD, Neumann AU, Perelson AS, Chen W, Leonard JM, et al. Rapid turnover of plasma virions and CD4 lymphocytes in HIV-1 infection. Nature. 1995;373:123-6.

7. Simon V, Ho DD. HIV-1 dynamics in vivo: implications for therapy. Nat Rev Microbiol. 2003;1:181-90.

8. Nowak MA, Anderson RM, Boerlijst MC, Bonhoeffer S, May RM, et al. HIV-1 evolution and disease progression. Science. 1996;274:1008-11.

9. Palella Jr FJ, Delaney KM, Moorman AC, Loveless MO, Fuhrer J, et al. Declining Morbidity and Mortality among Patients with Advanced Human Immunodeficiency Virus Infection. N Engl J Med. 1998;338:853-60.

10. Tran BX. Quality of Life Outcomes of Antiretroviral Treatment for HIV/AIDS Patients in Vietnam. PLoS ONE. 2012;7: e41062.

11. Tang SY, Xiao YN, Wang N, Wu H. Piecewise HIV virus dynamic model with CD4+ T cell count-guided therapy: I. J Theor Biol. 2012;308:123-34.

12. Hadjiandreou MM, Conejeros R, lan Wilson D. Long-term HIV dynamics subject to continuous therapy and structured treatment interruptions. Chem Eng Sci. 2009;64:1600-17.

13. Huang Y, Lu T. Modeling long-term longitudinal HIV dynamics with application to an AIDS clinical study. Ann Appl Stat. 2008:2:1384-408.

14. Smith RJ. Adherence to antiretroviral HIV drugs: how many doses can you miss before resistance emerges?. Proc Roy Soc B. 2006;273:617-24.

15. Verotta D. Models and estimation methods for clinicalHIV-1 data. J Comput Appl Math. 2005;184:275-300.

16. Wu H, Huang Y, Acosta EP, Rosenkranz SL, Kuritzkes DR, et al. Modeling long-term HIV dynamics and antiretroviral response: effects of drug potency, pharmacokinetics, adherence, and drug resistance. J Acquir Immune Defic Syndr. 2005:39:272-83.

17. Blower SM, Gershengorn HB, Grant RM. A tale of two futures: HIV and antiretroviral therapy in San Francisco. Science. 2000;287:650-4.

18. Huang Y, Rosenkranz SL, Wu H. Modelling HIV dynamics and antiviral response with consideration of time-varying drug exposures, adherence and phenotypic sensitivity. Math Biosci. 2003;184:165-86.

19. Nowak MA, May RM. Virus dynamics: mathematical principles of immunology and virology. Oxford: Oxford University Press; 2000.

20. Perelson AS, Neumann AU, Markowitz M, Leonard JM, Ho DD. HIV-1 dynamics in vivo: virion clearance rate, infected cell life-span, and viral generation time. Science. 1996;271:1582-6.

21. Perelson AS, Nelson PW. Mathematical analysis of HIV-1 dynamics in vivo. SIAM Rev. 1999;41:3-44

22. Abernethy RB. The New Weibull Handbook, 4th edition. Florida: Pratt \& Whitney Aircraft, Government Products Division United Yechnologies Corporation; 2000.

23. Nash FR. Estimating device reliability: assessment of credibility. Kluwer: Academic Publishers; 1993

24. Mills EJ, Bakanda C, Birungi J, Chan K, Ford N, et al. Life expectancy of persons receiving combination antiretroviral therapy in low-income countries: a cohort analysis from Uganda. Ann Intern Med. 2011;155:209-16. 
25. Collaboration ATC. Life expectancy of individuals on combination antiretroviral therapy in high-income countries: a collaborative analysis of 14 cohort studies. Lancet. 2008;372:293-9.

26. Johnson LF, Mossong J, Dorrington RE, Schomaker M, Hoffmann CJ, et al. Life expectancies of South African adults starting antiretroviral treatment: collaborative analysis of cohort studies. PLoS Med. 2013;10:e1001418.

27. May M, Gompels M, Delpech V, Porter K, Post F, et al. Impact of late diagnosis and treatment on LE in people with HIV-1: UK Collaborative HIV Cohort (UK CHIC) Study. BMJ. 2011;343:d6016.

28. Harrison KM, Song R, Zhang X. Life expectancy after HIV diagnosis based on national HIV surveillance data from 25states, United States. J Acquir Immune Defic Syndr. 2010;53:124-30.

29. Wu H, Zhang JT. The study of long-term HIV dynamics using semi-parametric non-linear mixed-effects models. Stat Med. 2002;21:3655-75.

30. Gabrielsson J, Weiner D. Pharmacokinetic and Pharmacodynamic Data Analysis: Concepts and Applications. Stockholm: Swedish Pharmaceutical Press; 2007.

31. Pantaleo G, Graziosi C, Fauci AS. Immunopathogenesis of human immunodeficiency virus infection. N Engl J Med. 1993;328:327-35.

32. Rosenberg ZF, Fauci AS. Immunopathogenic mechanisms of HIV infection. Clin Immunol Immunopathol. 1989;50: S149-56.

33. Willig JH, Abroms S, Westfall AO, Routman J, Adusumilli S, et al. Increased regimen durability in the era of once-daily fixed-dose combination antiretroviral therapy. AIDS. 2008;22:1951-60.

34. Li HP, Guo W, Li H, Wang Z, Liu YJ, et al. Occurrence of human immunodeficiency virus-1 resistance through a six year surveillance in rural areas of Henan. Natl Med J China. 2011;91:1443-7.

35. Xiao Y, Miao H, Tang S, Wu H. Modeling antiretroviral drug responses for HIV-1 infected patients using differential equation models. Adv Drug Del Rev. 2013;65:940-53.

36. Arora P, Dixit NM. Timing the emergence of resistance to anti-HIV drugs with large genetic barriers. PLoS Comput Biol. 2009;5:1000305.

37. Li J, Luo C, de Klerk N. Trends in infant/child mortality and life expectancy in indigenous populations in Yunnan Province, China. Aust NZ J Publ Heal. 2008;32:216-23.

\section{Submit your next manuscript to BioMed Central and we will help you at every step:}

- We accept pre-submission inquiries

- Our selector tool helps you to find the most relevant journal

- We provide round the clock customer support

- Convenient online submission

- Thorough peer review

- Inclusion in PubMed and all major indexing services

- Maximum visibility for your research 